Rev. salud pública. 14 sup (1): 43-55, 2012

Ensayos/Essays

\title{
La sociedad del riesgo y las inequidades en la salud de los trabajadores
}

\section{Risk Society and inequalities in the health of workers}

\author{
Silvia Tamez-González y Josué F. Pérez-Domínguez \\ Universidad Autónoma Metropolitana. Xochimilco. México.stamez@correo.xoc.unam.mx, josuefpd@ \\ gmail.com
}

Recibido 9 Octubre 2009/Enviado para Modificación 12 Diciembre 2010/Aceptado 5 Mayo 2011

\section{RESUMEN}

Reflexión sobre la situación de salud de los trabajadores, así como de las características que adquiere su cuidado dentro del contexto de un mundo globalizado. Para analizar este tema, se inicia con una discusión de los principales conceptos de globalización y sociedad del riesgo. En un segundo momento y bajo la perspectiva conceptual definida, se presenta un panorama estadístico de la salud de los trabajadores a nivel mundial, enfatizando la inequidad existente entre el mundo considerado desarrollado y el de los países pobres o en desarrollo. Posteriormente se discute acerca de los sistemas de salud y su incapacidad de dar respuesta eficiente a los problemas de salud de los trabajadores. Finalmente, se reflexiona en torno a la necesidad de replantear las estrategias de abordaje y de acción para contribuir a mejorar el estado de salud de los trabajadores y sus familias, poniendo en el centro el rescate de la "vida buena" y lo humano.

Palabras Clave: Globalización riesgo, salud, trabajadores, condiciones de trabajo, sociedad, riesgo (fuente: DeCS, BIREME)

\section{ABSTRACT}

This is a reflection on the current health situation of workers, as well as a reflection on the characteristics of their care system in the context of a globalized world. In order to present this reflection, the first part is focused on the discussion of the main concepts of globalization and risk society. On the second part, and according to the conceptual framework established on the first part, a statistical perspective of workers' health around the world is suggested, emphasizing on the existing inequity between thought-to-be developed world and the developing or poor countries. On the next part, a discussion related to health insurance systems and their incompetence to tackle efficiently workers' health outcomes is established. On the final part, a reflection on the need to reframe the approach and action strategies for improving health status of workers and their families is suggested; this part of the reflection is focused on the recovery of "good life" and human sense of life. 
Key Words: Globalization, health, workers, working conditions (source: $\mathrm{MeSH}$ )

- T ivimos en una sociedad global de riesgo? ¿La globalización ha globalizado los riesgos de salud? ¿Cuál es el papel del Estado $U_{\text {en }}$ en situación como ésta? ¿Cuál la posición de la medicina
social? ¿Cuáles las posibles soluciones a la crisis civilizatoria actual desde nuestra América?

Partiendo de las conclusiones de Edmundo Granda sobre el riesgo epidemiológico y su reflexión sobre la sociedad del riesgo, podemos afirmar que el mundo actual se caracteriza, hoy más que nunca, por la proliferación de riesgos sociales, políticos, ecológicos, económicos que "tienden cada vez más a escapar del monitoreo y protección de las instituciones" creadas para ello; y que éstas mismas instituciones "se constituyen en las productoras y legitimadoras de los peligros que no pueden controlar" (1). Bastaría analizar unos cuantos casos recientes para comprobar la certeza de esta afirmación.

Los riesgos a la vida, a la salud impuestos por la explotación capitalista siguen cobrando víctimas entre los mineros del mundo. En México una nueva explosión en el estado de Coahuila ha dejado otros dos muertos, producto de la negligencia y el afán de enriquecimiento de Minera México, que se suman a los 65 mineros que murieron aplastados en el derrumbe de la mina en Pasta de Conchos $(2,3)$.

La reciente epidemia de influenza A H1N1 nos muestra cómo la acumulación capitalista no repara en las consecuencias que para la vida en general conlleva la utilización cada vez más intensiva de tecnología y la utilización irracional de los descubrimientos científicos en la genómica, como demostraría (en caso de que se verificara), la hipótesis de que el virus se generó en las granjas de explotación porcina mexicanas filiales de la Smithfield Foods Inc (4). Riesgos a la salud de la especie humana que no encuentran una respuesta ético-política, sino técnico-económica; es decir, en vez de que los gobiernos del mundo y la OMS se avoquen a crear sistemas únicos de salud que garanticen el acceso universal de la población mundial a la misma, se dedican a destruir y a privatizar los restos de los sistemas públicos de salud, favoreciendo de esta manera el dominio aplastante de las grandes empresas farmacéuticas y aseguradoras privadas que han hecho de la vida y la muerte, de la salud y la enfermedad, un negocio redondo. 
Para controlar la expansión de la epidemia, los gobiernos del mundo, en especial el de México, así como la OMS, recurrieron a medidas autoritarias basadas en consideraciones tecnocráticas, sin la suficiente fundamentación epidemiológica. De esta manera, se canceló la posibilidad de cualquier respuesta crítica organizada por parte de la sociedad civil pues los medios de comunicación masiva se encargaron de mantenerla en estado de shock y se reservaron el derecho de cerrar los espacios públicos de discusión, análisis y organización social, estableciendo de facto un virtual estado de sitio, al tiempo que se autoproclamaban como "salvadores de la humanidad" (5).

Entonces, junto a la "sociedad del riesgo" de Bech (6), tendríamos la "sociedad sitiada" de Bauman (7). Esto quiere decir que, si bien, con la mundialización de los procesos productivos y cambiarios capitalistas se generalizan los riesgos sociales, económicos, políticos, etc., incluidos los de salud, el poder del Estado no desaparece, sino se refuncionaliza: de ser garante de las condiciones políticas, sociales y materiales de la reproducción del capital a nivel nacional, se convierte en agente que intermedia por las grandes corporaciones trasnacionales, encargado de la "seguridad nacional", y por lo tanto, despojado de toda visión e intención integradora y/o conciliadora, quedándose en el puro esqueleto: las funciones represivas y de control social.

Frente a una situación como ésta el reto de la ciencia en general, y de la medicina social y la salud pública en particular, es desarrollar una nueva práctica liberadora, que, siguiendo a Granda, parta de un presupuesto filosófico-teórico de la salud y la vida, con métodos capaces de integrar y comprender lo diverso, lo temporal y lo complejo, y comprometida con los procesos, actores y movimientos sociales que luchan por el derecho a la salud, la democratización de la toma de decisiones, y un nuevo proyecto civilizatorio no-capitalista (o un rumbo no-capitalista de la civilización).

Globalización de los riesgos y crisis capitalista

Si partimos de la distinción que hace Ulrich Bech entre globalización y globalismo, entendida la primera como un proceso de imbricación mundial, multidimensional, policéntrico y contingente, y el segundo como una reducción economicista de la primera, tenemos que durante los últimas décadas, en torno a este proceso multidimensional se ha elaborado un discurso legitimador de las imposiciones políticas, económicas y militares 
de los Estados Unidos. Estas imposiciones amenazan con destruir, no sólo los logros humanos por establecer una vida social digna, sino hasta la propia vida en general, antes que desaparecer como potencia hegemónica en decadencia. Este discurso, conocido comúnmente como neoliberalismo, con pretensiones de cientificidad, ha sido utilizado y aplicado sus postulados en prácticamente la totalidad de los países de nuestra América. Sin embargo, la realidad esencialmente contradictoria del capitalismo, así como las luchas de resistencia y los gobiernos contra-hegemónicos a lo largo de nuestra geografía, han provocado su rotundo fracaso y han demostrado la falacia de sus planteamientos bajo los mantos de la "objetividad científica".

Lo que se ha dado en las últimas décadas no es tanto una integración global, sino integraciones regionales, en donde las distintas corporaciones y sus gobiernos buscan ventajas comparativas en sus intercambios y tratados con otras regiones económicas. Ejemplo de lo anterior, es la existencia de las zonas maquiladoras en la frontera entre México y Estados Unidos y a lo largo de toda Centroamérica.

El sistema-mundo capitalista no tiende pues, a una integración armónica y equilibrada entre "los países desarrollados y los países en vías de desarrollo" (como si desde el origen mismo de este sistema-mundo no existiera contradicción alguna entre unos y otros), ni si quiera a la existencia un solo centro mundial y diversas periferias y semi-periferias, sino a una reproducción del "orden mundial" policéntrica y multidimensional, como observa Bech, pero "altamente caótica", según Wallerstein (8).

La reconfiguración del mundo llevada a cabo por el capitalismo en los últimos 30 años ha redefinido así mismo los riesgos en salud. La llamada revolución informática y las sucesivas crisis y sus efectos mundiales, han extendido el desempleo y la fragmentación de la fuerza de trabajo en nuevas categorías: trabajadores informales, precarios, auto-empleados, migrantes, forzados, etc. Estas nuevas categorías de trabajadores están casi completamente desamparados frente a las prácticas laborales de explotación de las grandes corporaciones capitalistas.

En el México de fines del siglo XIX y principios del XX, la figura del "enganchador" funcionaba como primer contratista de campesinos, mujeres, niños e indígenas que después "vendía" a los patrones de las haciendas como mano de obra semi-esclava. Actualmente el fenómeno de 
la subcontratación (o "outsourcing") funciona como moderno mecanismo de "enganchamiento" de una fuerza de trabajo despojada de todo derecho a la libre asociación o sindicalización. A los trabajadores, principalmente jóvenes, subcontratados para las empresas de alimentos como Burger King, Domino's Pizza, Starbucks Coffee, etc., se les explota obligándolos a trabajar más horas de las establecidas en el contrato individual para luego "pagárselas con horas"; es decir, en vez de pagarles las horas extras trabajadas, los mandan a su casa uno o dos días que obviamente, al no ser trabajados, no son pagados. Si a esto agregamos que de los "1,1 billones de jóvenes de entre 14 y 25 años en todo el mundo, 1 de cada 3 anda en busca pero no encuentra empleo", nos encontramos con un panorama en el que los jóvenes son no sólo sujetos de riesgo, sino también de estigmatización y criminalización.

La sociedad del riesgo, el trabajo y la salud

El informe de la EMCONET (Employment Conditions Knowledge Network) titulado "Condiciones de Empleo e Inequidades en Salud" de la Comisión de Determinantes Sociales de Salud (CDSS) de la Organización Mundial de la Salud, realmente constituye uno de los documentos más completos sobre el tema, pues analiza los daños a la salud desde dos perspectivas. La primera, más general, señala la centralidad del trabajo en la configuración de la clase social, y por lo tanto, fundamental para la definición de los perfiles de salud. La segunda, más concreta, analiza aspectos directamente relacionados con las condiciones específicas de los lugares de trabajo, posibilitando así la construcción de una visión completa e integral del problema.

En el primer caso, cuando se analiza la información disponible sobre las diferentes situaciones de empleo en el pasado, encontramos que Marie Jahoda observó en Canadá que la desnutrición, el bajo peso al nacer, la enfermedad cardiovascular y la ansiedad se presentan con mucha mayor frecuencia en los desempleados. Así mismo, algunos autores han mostrado que la enfermedad mental en hombres desempleados es significativamente mayor que en los empleados.

En cuanto a los problemas de salud generados en el sitio de trabajo hay que tener en cuenta que cuando hablamos de la salud de los trabajadores, nos estamos refiriendo a una población de más de tres mil millones de 
personas que constituyen alrededor de la mitad de la población mundial. Existen estudios que muestran que alrededor de la mitad de estos tres mil millones se exponen a altos niveles de riesgos ocupacionales.

¿Cómo se traduce este hecho en la salud? Algunas evaluaciones reportan que se registran al año 264 millones de eventos que causan al menos tres días de incapacidad, es decir 700000 trabajadores lesionados por día. Respecto a los eventos fatales, existen estimaciones que señalan que anualmente ocurren 350000 accidentes fatales, lo que significa que todos los días mueren en el mundo 970 trabajadores por esta causa. Más aún, las muertes relacionadas con el trabajo, incluyendo accidentes pero también aquellas causadas por cáncer, enfermedad cardiovascular y otras enfermedades ascienden a dos millones anualmente, es decir, cada día, mueren 5000 trabajadores a causa de su trabajo. De estas muertes, las principales causas son accidentes con el $41 \%$, seguidas por Enfermedad Pulmonar Obstructiva Crónica (EPOC) con $40 \%$ y cáncer de tráquea, bronquios o pulmones con el $13 \%$, que en conjunto, se traducen en 22 millones de años de vida saludable perdidos. A su vez, Concha-Barrientos ha calculado que en total, las lesiones fatales y no fatales del trabajo representan el 8,8\% de la carga global de mortalidad.

En cuanto a la distribución geográfica de este fenómeno existe algunas reveladoras estimaciones del Banco Mundial que indican que en China y la India el riesgo de lesiones fatales y no fatales a causa del trabajo, es dos y media veces más alta que en Europa y Norteamérica. Además, esta diferencia es cinco veces más alta en el caso de África Sub-Sahariana.

Así mismo, los países ricos tienen las tasas más bajas. Por ejemplo, Suecia y el Reino Unido reportan respectivamente una tasa de lesiones fatales de 1,9 y 0,8 por cada 100000 trabajadores, mientras que en Mozambique, Kenya y Bolivia, estos eventos pueden alcanzar una tasa de hasta 21.9 por 100,000 trabajadores. Otros ejemplos reveladores en relación a la distribución en función del nivel de desarrollo de los países, muestran que en México las lesiones fatales son tres veces más altas que en Estados Unidos (15,9 vs 5,2). Comparando Marruecos y España las diferencias son aún más dramáticas registrando una tasa de lesiones fatales de 47,8 por 100 000 trabajadores en el primer país contra 8,9 en el segundo, es decir, una diferencia 5 veces mayor. 
Desde luego, los costos económicos que representan para la sociedad estos riesgos son muy altos. Así tenemos que los costos por compensaciones, pérdida de tiempo, interrupción de la producción, capacitación y gastos médicos representan el $4 \%$ del Producto Interno Bruto global anual, lo que en 2001 representó la exorbitante suma de mil doscientos cincuenta billones de dólares, veinte veces mayor que la asistencia oficial al desarrollo.

Por su parte, desde la perspectiva de las enfermedades de trabajo el panorama tampoco es muy alentador. Es sabido que tanto en países desarrollados como no desarrollados, millones de trabajadores se exponen a miles de substancias químicas, cientos de agentes biológicos y docenas de factores físicos con efectos importantes para su salud (9). Al respecto, se tiene que reconocer que, aún en países como Estados Unidos y algunos de la Unión Europea, existe una fuerte necesidad de prevenir las enfermedades profesionales causadas por factores físicos, químicos o biológicos.

Así tenemos que, por ejemplo, en España se calcula que el 25,4 \% de los trabajadores están expuestos a carcinógenos, lo que puede ascender al $52 \%$ en los sectores más peligrosos. En este país, también se reportaron cerca de 30000 enfermedades laborales en 2004 pero otros análisis más precisos han estimado que realmente se producen 80000 enfermedades de trabajo anualmente, de las cuales, 16000 son fatales.

Por su parte, datos de la OMS señalan que un cuarto de la fuerza de trabajo de los países desarrollados y poco más de tres cuartos en los países pobres está expuesta a alto riesgo por exposición a factores físicos (maquinaria en movimiento, ruido, vibraciones radiaciones ionizantes y no ionizantes, polvos, etc.) y prácticamente el total de los trabajadores de la minería y la construcción. Destaca entre los riesgos físicos, las fibras de asbesto, pues se calcula que más de 125 millones se exponen a esta fibra en su lugar de trabajo y por lo menos se producen 90000 muertes cada año por cáncer pulmonar, mesotelioma y asbestosis resultantes de la exposición profesional (10).

En fin, sería posible seguir enunciando evidencias sobre la magnitud del problema de salud de los trabajadores y sus familias, sin embargo, creemos que los datos arriba presentados reflejan su complejidad. 
La seguridad social de los trabajadores en un mundo en crisis

La paulatina destrucción de los sistemas de protección social auspiciada desde el Banco Mundial, la OMC, los gobiernos neoliberales (incluidos los partidos socialdemócratas y laboristas), ha puesto a la mayor parte de la clase trabajadora a un nivel de vulnerabilidad y explotación similar al existente en el último cuarto del siglo XIX. Los 1,37 billones de trabajadores pobres que viven con 2 dólares al día, de los cuales $60 \%$ son mujeres, se han convertido en un problema impostergable para los gobiernos y organismos del mundo. Sin embargo, las soluciones emprendidas no pasan de programas asistencialistas (en México el llamado Oportunidades, que pretende en el discurso poner a las familias pobres en condición de igualdad con las familias "no-pobres" para que puedan enfrentarse a los avatares del mercado), así como de planes de desarrollo de mediano plazo que incluyen la creación de instituciones de protección a la salud endebles y dependientes de la infraestructura y personal médico del viejo y deteriorado sistema de seguridad social. Ejemplo de lo anterior lo encontramos en México, con el llamado Seguro Popular de Salud, ideado por Frenk, que pretende asegurar a los trabajadores informales y precarios en base al pre-pago de una prima $\mathrm{y}$ a las aportaciones proporcionales del Estado y cubriendo un abanico muy limitado de daños a la salud (11).

Por otro lado, a pesar de lo completo y detallado del informe de la CDSS de la OMS, desgraciadamente sus investigadores no contaron con las últimas cifras de desempleo abierto en el mundo. La actual crisis económica mundial ha arrojado a millones de trabajadores a la calle y hacia el umbral de miseria. Si la tasa de desempleo en la mayor economía del mundo, los Estados Unidos, es superior al $9 \%$ y a pesar de los buenos augurios de sus economistas, no se perciben visos de recuperación, podemos cuestionarnos si la sociedad global del riesgo no estará más bien transitando hacia una sociedad global en crisis total y permanente.

Como algunos analistas han argumentado, la crisis económica, ecológica, alimentaria y energética que padece el planeta demostraría, que estamos ante una crisis estructural del capitalismo, y más aún, ante una profunda crisis civilizatoria en la que el mito de la ciencia, la tecnología y el progreso, lejos de garantizar el bienestar y felicidad de la humanidad devienen en principales amenazas de la misma. 
Si en los primeros años de los noventa, cuando las recomendaciones del FMI y del Banco Mundial se comenzaron a aplicar autoritariamente en los distintos países que abrazaron el neoliberalismo como tabla salvadora a la crisis fiscal y de la deuda de los Estados, la probabilidad de quedar desempleado o de ver reducidos los derechos sociales de los trabajadores era un "riesgo", actualmente, cuando la crisis del sistema capitalista se ha profundizado y asemeja a la Gran Depresión de 1929, el desempleo, el trabajo informal y precario, e incluso, el trabajo forzado e infantil, no pueden ser considerados un riesgo sino una situación naturalizada socialmente para los países pobres. Así, para la mayoría de los seres humanos que habitan los países de África, América Latina y Asia, más que un riesgo, es una realidad crítica y desesperada que se alarga ya por varias décadas.

¿Cómo hablar de "riesgos" como lo hace Soberanes (el ombudsman mexicano), al decir que "en 2009, el riesgo de fallecer en la región fronteriza entre México y Estados Unidos es 1,5 veces más alto que en 2004 y 17 veces mayor que en 1998" (12), sin criticar la política económica impuesta por el TLC que destruye la producción agrícola, el tejido social y la cultura de cientos de comunidades campesinas e indígenas provocando año con año la migración de miles de mexicanos y centroamericanos? Los 5 mil 600 muertos de los últimos 15 años al intentar cruzar "al otro lado" no deberían señalarnos solamente el "mayor riesgo" de muerte del migrante, sino la catastrófica realidad de millones de personas, pertenecientes a pueblos y comunidades que no están "en vías de desarrollo", sino en vías de desaparición.

La imposibilidad de incrementar sus ganancias a complacencia desde la crisis de 1973, lo que significa un estancamiento de la acumulación de capital a nivel mundial, es lo que ha llevado a las grandes corporaciones y sus sirvientes en los gobiernos a modificar las legislaciones y relaciones laborales, con el propósito de facilitar la reconversión productiva hacia una mayor flexibilización, no sólo tecnológica, sino también laboral. Las grandes corporaciones capitalistas no sólo exportan su capital y lo invierten en zonas donde pagarán bajos salarios, sino que además dentro de sus propios países de origen incrementan el grado de explotación de la fuerza de trabajo destruyendo las jornadas completas y las contrataciones colectivas, sustituyéndolas por el trabajo temporal y por horas.

Además, la capacidad de negociación de los sindicatos ha sido debilitada tanto por los instrumentos y reformas legales, como mediante la represión 
directa. Ante esta ofensiva de las grandes corporaciones capitalistas contra los trabajadores de todo el mundo, el Estado no sólo no defiende a las grandes mayorías (como supondrían los principios democráticos) sino que edifica la ficción de la protección social mediante esquemas de seguridad social y de salud que sólo benefician a la industria farmacéutica y a las aseguradoras privadas. Sistemas privados de protección social, como las AFORE en México, que permiten la especulación financiera por parte de las aseguradoras y la utilización por parte de los gobiernos de los fondos de pensión de los trabajadores como "cajas chicas" ante las emergencias de la crisis y su situación de insolvencia.

Si como muestran diversos estudios, entre ellos el de la CDSS de la OMS, el desempleo, el trabajo informal y precario no solamente se dan en los países periféricos y semi-periféricos, sino también y de manera cada vez más extensa, en los países desarrollados de Europa y en Estados Unidos, la promesa del crecimiento y posterior distribución equitativa de la riqueza producida mediante el incremento de la productividad y el progreso tecnológico se une a las ficciones del pleno empleo y del empleo justo (o fair employment).

En el capitalismo no puede haber relación laboral justa, pues ésta descansa sobre la explotación del trabajo vivo por parte del capital y sobre la existencia de una población excedente que sirve, como decían los clásicos, de "ejército industrial de reserva" para satisfacer las necesidades de la acumulación capitalista. El empleo justo y el crecimiento económico para todos son otras tantas de las ficciones de la economía burguesa utilizadas para asegurar la continuidad de la dictadura del capital.

Desde esta perspectiva es que podemos entender las reformas a los sistemas de salud, de seguridad social y laborales realizadas (y las aún por realizar) en nuestra América en los últimos años. Es decir, para el capital internacional, es cuestión de vida o muerte apropiarse no sólo del tiempo de trabajo excedente extraído mediante la explotación de la fuerza de trabajo, sino también del tiempo de trabajo necesario para la propia reproducción del obrero, aunque eso suponga el acortamiento del tiempo de vida útil del mismo (como registra el informe de la CDSS de la OMS: cada año 1 000 trabajadores en el mundo pierden 3,5 años de vida saludable debido a accidentes de trabajo). 
La cuestión del Estado y la "vida buena"

Las necesidades humanas están completa y como nunca subordinadas a las necesidades del capital. Es una lucha de vida o muerte. En esta lucha, nuestra práctica médica y científica tiene que optar, contra las tendencias ideológicas dominantes, a favor de la vida. Pero no de la vida tal como está en el capitalismo, una vida subordinada y enferma, sino una "vida buena", entendida como la vida libre y activa que ya desde Aristóteles era considerada como el fin de la vida social, como el sumak kawsay de la nueva Constitución de Ecuador y el teko kavi de la nueva Constitución de Bolivia. Ambos conceptos aparecen en las respectivas constituciones políticas aprobadas recientemente en esos países y representan la cosmovisión kichwa, aymara y demás pueblos indígenas, donde lo humano se encuentra en armonía con lo otro: la naturaleza y sus fuerzas.

Una vida buena, digna, no sólo es aquella donde el trabajador es respetado por sus superiores, recibe un ingreso adecuado a sus necesidades, su actividad es enriquecedora para él mismo, tiene acceso a la atención médica, y demás características del "fair employment" de la CDSS de la OMS, que según sus investigadores debería ser elevado a derecho humano, seguramente para que se agregara a la lista de derechos humanos violados sistemáticamente por todos los gobiernos del mundo; la vida buena es aquella en donde el mismo estatuto de "trabajador" queda superado por la recuperación de la cualidad humana de la persona (y no precisamente mediante la promulgación protocolaria de buenos deseos convertidos en derechos). No una vida subordinada por y para el trabajo, sino la actividad social reproductora de la vida al servicio de la dignidad y plenitud humanas. Esto supone, por supuesto, la desaparición del trabajo asalariado y la existencia misma del capital. Todo trabajo asalariado, como afirma Marx, es trabajo forzado, pues responde a una necesidad ajena al propio trabajo. "Su carácter ajeno lo pone de relieve el hecho de que, tan pronto deja de existir alguna coacción física o de cualquier otro tipo, se huye del trabajo como de la peste" (13).

Con este horizonte en mente, y tomando en cuenta la crisis del mito de la ciencia y su capacidad emancipadora, la cuestión sobre la fundamentación de nuestra práctica científica adquiere principal relevancia. Si partimos de que la salud no es la "ausencia de enfermedad" en el cuerpo, sino 
el resultado siempre precario de múltiples determinaciones sociales, económicas, políticas, culturales, etc., que permiten o impiden al individuo y las sociedades auto-realizarse como humanidad, entonces podemos "descentrar" la lucha por el derecho a la salud del papel del Estado.

Es decir, centrar la resolución de los problemas de salud en la capacidad o incapacidad del Estado para brindar servicios de salud equitativos y de calidad, es limitar nuestra perspectiva teórica y práctica no sólo de la posible solución de los mismos, sino de nuestra misma concepción de salud. Si pensamos que sólo el Estado, por su capacidad centralizadora y al mismo tiempo omnipresente en todos los ámbitos de la vida, puede brindar servicios de salud, nos encerramos en la idea de que la salud es evitar o acabar con las enfermedades, y por lo tanto, nuestra práctica médica y científica, comprometida de por sí con el derecho a la salud de nuestros pueblos, quedará limitada, centrada, y constantemente frustrada, ante la imposibilidad de "reformar" o "democratizar" al Estado capitalista.

Por lo menos en lo que respecta a México, democratizar al Estado, con los instrumentos políticos reconocidos por ese mismo Estado, es una posibilidad que la realidad una y otra vez ha anulado. Desde el movimiento estudiantil de 1968, pasando por la "guerra sucia" de la década de 1970 hasta el último fraude electoral de 2006, el Estado mexicano ha recurrido una y otra, y cada vez más, a la represión. Estamos de acuerdo con el postulado del Movimiento de Reforma Sanitaria de Brasil de que la lucha por el derecho a la salud forma parte de una lucha más amplia por la democratización del Estado. Sin embargo, la presente crisis estructural del capitalismo muestra que la tendencia general no es hacia la democratización del Estado sino hacia el reforzamiento de las funciones represivas, defensivas y ofensivas. De ahí el alto gasto militar del gobierno de Lula, su intervención en Haití, el paramilitarismo de Uribe en Colombia, la represión genocida de García contra los pueblos indígenas del Perú, la guerra contra el crimen organizado y la criminalización de la lucha social por parte del gobierno de Calderón en México.

El proceso político en Bolivia sería tal vez el único caso ejemplar de democratización del Estado desde abajo. Son los pueblos indígenas, campesinos, obreros, estudiantes, quienes en abierta confrontación contra las oligarquías locales, llevaron a uno de los suyos al poder del Estado. La refundación democrática y radical del Estado, le permite al pueblo 
boliviano constituirse en poseedor y garante del usufructo de sus recursos naturales, como el agua, el gas y el litio, tan ambicionados por las grandes corporaciones capitalistas.

Por tal razón, la lucha por el derecho a la salud de nuestros pueblos no puede disociarse de la lucha por la liberación contra la dominación estadounidense. La propia concepción de salud no puede disociarse de la "vida buena", y ambas sólo pueden volverse una realidad mediante la democratización de las decisiones políticas fundamentales que afectan la vida colectiva, no necesariamente centradas en el Estado, sino impulsadas por y desde los movimientos sociales y populares, que al ir construyendo espacios y formas de participación política, no sólo van confrontando los aparatos represivos y de control de los Estados nacionales y las formas de dominación del capital internacionalizado (incluidos los programas asistencialistas de atenuación de la pobreza), sino que van construyendo, así mismo, nuevas relaciones sociales que son la simiente de una vida buena y saludable

\section{REFERENCIAS}

1. Granda E. Globalización de los riesgos en salud [internet]. Ponencia presentada en el IV Congreso Internacional de Salud Pública "Globalización, Estado y Salud", realizado en la Universidad de Antioquía, 10, 11 y 12 de noviembre, Colombia, 2005. Disponible en: http://www.alames.org/documentos/globalizacion.pdf. Consultado Octubre de 2009.

2. La Jornada (Periódico diario), Edición: 7 de agosto de 2009.

3. Fernández J, Rajchenberg E, Tamez S, Amparo G. La explosión en pasta de conchos: Una historia repetida. Lux. 2006; 559-560: 98-101.

4. Hernández L. Smithfield: un negocio muy marrano. La Jornada (diario); 5 de mayo de 2009.

5. Hernández L. Fin de época. La Jornada (diario); 19 de mayo de 2009.

6. Bech U. ¿Qué es la globalización? Barcelona: Paidós; 1998.

7. Bauman Z. La sociedad sitiada. México: Fondo de Cultura Económica; 1992.

8. Wallerstein I. La depresión: una visión a largo plazo. La Jornada (diario); 19 de octubre de 2008.

9. Benach J, Muntaner C, Santana V. Employment Conditions and Health Inequalities. Employment Conditions Knowledge Network (EMCONET); 2007.

10. WHO, 2006 [Internet]. Disponible en: http://www.who.int/whr/2006/en/ . Consultado: Octubre de 2009.

11. Tamez S, Eibenschutz C. El Seguro Popular en Salud: pieza clave de la inequidad en salud. Rev. salud pública (Bogotá) 2008; 10 sup (1): 133-145.

12. Olivares E. Muere un mexicano cada día al intentar llegar a EU: $\mathrm{CNDH}$. [Internet] Disponible en http://www.jornada.unam.mx/2009/09/25/politica/019n1pol Consultado 5 de octubre de 2009.

13. Fromm E. Marx y su concepto del hombre. Incluye: "Manuscritos económico-filosóficos" de Marx K. México: FCE; 1962. 